\title{
O Efeito do Estresse na Qualidade de Vida de Idosos: O Papel Moderador do Sentido de Vida
}

\author{
The Effect of Stress on Elderly Quality of Life: Meaning in Life \\ as a Moderating Role
}

\author{
Rômulo Lustosa Pimenteira de Melo*, ${ }^{*}$, Maria do Carmo Eulálio ${ }^{b}$, Valdiney Veloso Gouveia ${ }^{a}$ \\ \& Hermesson Daniel Medeiros Silva ${ }^{a}$ \\ ${ }^{a}$ Universidade Federal da Paraíba, João Pessoa, Brasil \& ${ }^{b}$ Universidade Estadual da Paraíba, João Pessoa, Brasil
}

\begin{abstract}
Resumo
O objetivo principal deste estudo foi conhecer se o sentido de vida pode moderar a relação entre o estresse e a qualidade de vida em idosos. Participaram 210 pessoas com idade média de 74 anos, a maioria do sexo feminino (68,58\%). Estas responderam aos seguintes instrumentos: Mini-Exame do Estado Mental, Inventário de Eventos de Vida Estressantes para Idosos, Questionário de Qualidade de Vida para Idosos, Teste de Propósito de Vida e perguntas demográficas. Foram realizadas análises de componentes principais, correlações de Pearson e regressões lineares. Os resultados indicaram que a influência do estresse na qualidade de vida era significativa para o grupo com baixo sentido de vida, porém não ocorreu o mesmo para o grupo com alto sentido de vida. Assim, o sentido de vida parece ter operado como um recurso protetor da qualidade de vida frente ao estresse, indicando seu papel moderador.

Palavras-chave: Sentido de vida, estresse, qualidade de vida, saúde, idoso.
\end{abstract}

\begin{abstract}
The main objective of this study has been to know if the relationship between stress and elderly quality of life can be moderated by the meaning in life. Two hundred and ten participants with mean age of seventy four years old, most of them female $(68.58 \%)$ took part in the study. The participants answered the following instruments: Mini Mental State Exam, Stressing Events Inventory, Elderly Quality of Life Questionnaire, Purpose in Life Test (PIL) and demographic questions. Principal component analysis, Pearson correlation coefficients and linear regressions were analyzed. The results obtained indicated that the influence of stress on quality of life is significant for the group with low meaning in life, but it was not the same for the group with high meaning in life. Thus, the meaning in life seems to have acted as a protective factor for the quality of life showing its moderating role with respect to stress.

Keywords: Meaning in life, stress, quality of life, health, elderly.
\end{abstract}

É notório o crescimento da população idosa no contexto brasileiro (Banco Internacional para a Reconstrução e o Desenvolvimento [BIRD], 2011), demandando maior conhecimento nas várias áreas do saber. Assim sendo, existe interesse em compreender não apenas os antecedentes de uma vida longa ou maior esperança de vida, mas também como envelhecer com a qualidade de vida satisfatória, apesar das diversidades cotidianas.

O processo de envelhecimento humano tem sido relacionado a mudanças de ordem biopsicossocial, que apresentam um efeito significativo na redução da saúde física e mental, diminuindo a qualidade de vida (Fortes-Burgos, Neri, \& Cupertino 2008). Tais mudanças, vivenciadas pelos idosos, corroboram o sentimento de perda de controle sobre si e sobre o ambiente, sendo comumente percebidas como potencialmente estressantes (Neri \& Fortes, 2006). A propósito, segundo Aldwin (1990; Aldwin \& Gilmer, 2004), os eventos estressantes do envelhecimento podem ser diferenciados em dois tipos básicos: egocêntrico e não-egocêntrico. O primeiro é fruto de eventos e preocupações que dizem respeito ao próprio idoso (por exemplo, problemas ligados a sua dependência física e sua condição econômica), já o segundo diz respeito a eventos ocorridos com pessoas significativas para o idoso (por exemplo, problemas de saúde e morte de parentes e amigos).

Apesar de apresentar uma classificação e compreensão geral, é preciso assinalar que nem todas as pessoas vivenciam o evento estressante da mesma forma; isso dependerá da avaliação que elas farão do evento em si. Quanto mais 
o evento for percebido como adverso e relacionado com a perda e a incontrolabilidade, maior a chance de serem vividos como estressantes (Monat, Lazarus, \& Reevy, 2007). Assim, um determinado agente estressor pode ser estressante para um idoso, mas não para outro. Esta distinção do potencial danoso do evento estressante se deve, em parte, à possibilidade de a pessoa utilizar recursos psicossociais que favoreçam estratégias de enfrentamento (coping) diante dos eventos (Lazarus \& Folkman, 1984; Margis, Picon, Cosner, \& Silveira, 2003). Neste marco, o sentido de vida (SV) poderia ser considerado um fator que auxilia no enfrentamento de situações consideradas adversas, potencializando os níveis de saúde e de qualidade de vida das pessoas.

Enquanto construto psicológico, o SV foi primeiramente tratado por Frankl (1978), que o concebia como um dos motivadores principais do ser humano. Sua abordagem, conhecida como logoterapia, sugere que a presença de SV está ligada ao bem-estar psicológico e subjetivo, enquanto que a sua ausência é capaz de gerar um estado de apatia e desânimo (Frankl, 2003, 2004). Depois deste autor, outros se dedicaram a avaliar o SV e seu efeito na saúde das pessoas. Por exemplo, tem sido demonstrado que este construto é um preditor importante de saúde física (Jim \& Andersen, 2007; Krause, 2009), bem-estar psicológico e qualidade de vida (Bauer-Wu \& Farran, 2005; Ho, Cheung, \& Cheung, 2010; Kashdan \& Steger, 2007; Melton \& Schulenberg, 2008; Park, Malone, Suresh, Bliss, \& Rosen, 2008; Steger \& Frazier, 2005). Contrariamente, sua ausência tem sido relacionada com níveis maiores de depressão, ansiedade (Mascaro \& Rosen, 2005; Steger, Mann, Michels, \& Cooper, 2009) e ideação suicida (Aquino, 2009).

Segundo Steger et al. (2009), Frankl não se preocupou em delimitar uma definição do construto SV, porém foi enfático em sugerir que ele estaria ligado à crença de que a vida da pessoa é plena de significados, que transcendem a própria realidade material. De acordo com Reker (1997), o SV está associado com um propósito, uma direção e uma razão para a existência. Ele é um construto multidimensional, apresentando componentes cognitivos, motivacionais e afetivos (Reker \& Wong, 1988). O cognitivo está relacionado com as crenças e interpretações do mundo; o motivacional diz respeito ao sistema de valores de cada indivíduo, os quais interferem na realização de metas pessoais; e, finalmente, o afetivo compreende a satisfação com a vida, isto é, a concepção de que a vida vale a pena.

O conhecimento de como o SV atua na saúde física e mental das pessoas é escassamente discutido, existindo menos ainda evidências empíricas. A propósito, McKnight e Kashdan (2009) sugerem que o sentido de vida atua como mecanismo de autorregulação, auxiliando na percepção de eventos e oferecendo uma sensação de significado existencial. Estes autores entendem que este construto, por si só, não regula o comportamento, porém direciona as pessoas para que, por meio da utilização de seus recursos psicossociais, possam superar com menos dificuldades os eventos estressores do dia a dia. Neste contexto, o presente estudo tem como objetivo conhecer em que medida o sentido de vida pode diminuir os efeitos do estresse na qualidade de vida de idosos.

\section{Método}

\section{Participantes}

Este artigo faz parte de um acompanhamento da amostra utilizada pela Rede FIBRA Assim, tomou-se como referência uma amostra composta por 254 idosos os quais apresentavam desempenho cognitivo acima da nota de corte ajustada para escolaridade no MEEM. Após um ano deste estudo, foram encontrados os endereços de 229 idosos, dos quais seis já haviam falecido e 13 começaram a apresentar déficit cognitivo. Neste sentido, já não poderiam fazer parte do estudo. Portanto, a amostra final foi composta por 210 idosos de Campina Grande (PB). A maioria do sexo feminino $(51,9 \%)$ com idade média de 74 anos $(D P=7,70)$, sendo que a maior parte $(51,90 \%)$ está no grupo de $70-79$. Quanto ao estado civil, $47,14 \%$ declararam ser casados /conviventes, com $28,1 \%$ de analfabetos e $58,6 \%$ que possuíam até os estudos básicos. A média de renda pessoal foi de 868 reais $(D P=1.100,00)$ com uma moda de 510 (55,50\% da amostra); 61,90\% dos idosos recebiam entre 0.0-1.0 salários mínimos, disso deriva a grande dispersão da renda pessoal dos idosos (os dados demográficos estão descritos na Tabela 1).

\section{Instrumentos}

Os participantes responderam a um protocolo composto pelos seguintes instrumentos:

Questionário de Qualidade de Vida para Idosos (WHOQOL-OLD). Este tem como objetivo mensurar a satisfação do idoso com os diversos aspectos que envolvem sua vida (Fleck, Chachamovich, \& Trentin, 2006). Sendo assim, a escala é composta por 24 itens, distribuídos em seis facetas com quatro itens cada uma: funcionamento dos sentidos, autonomia, atividades passadas, presentes e futuras, participação social, morte e morrer e intimidade. Os itens são respondidos em escala de cinco pontos, variando de 1 (Nada) a 5 (Completamente), segundo o conteúdo abordado (por exemplo, satisfação, felicidade). As pontuações são padronizadas, variando de 0 a 100; o somatório dos itens das seis facetas produz uma pontuação total de qualidade de vida.

Teste de Propósito de Vida (PilTest-12). Originalmente desenvolvido por Crumbaugh e Maholick (1964), este instrumento faz uma avaliação geral da sensação de realização existencial vivenciada. Sua versão inicial era composta por 20 itens, reunindo evidências de validade e precisão em diversos países; por exemplo, apresentou alfas de Cronbach entre 0,70 e 0,90 (Melton \& Schulenberg, 2008; Schulenberg \& Melton, 2010). Não obstante, emprega-se neste estudo a versão brasileira abreviada, composta de 12 itens, que são respondidos em escala 
Tabela 1

Perfil Sociodemográfico

\begin{tabular}{lllll}
\hline Variável & \multicolumn{3}{c}{ Categoria } \\
\hline \multirow{2}{*}{ Sexo } & Masculino & Feminino & \\
& $66(31,42 \%)$ & $144(68,58 \%)$ & \\
Grupos de idade & $65-69$ & $70-79$ & 40 \\
& $57(27,10 \%)$ & $109(51,90 \%)$ & & \\
Estado civil & Casado/convivente & Não casado & & \\
& $99(47,14 \%)$ & $109(52,86 \%)$ & & Est. Superiores \\
Escolaridade & Analfabeto & Est. Básicos & Est. Médios & \\
& $59(28,1 \%)$ & $123(58,6 \%)$ & $19(9 \%)$ & \\
Atividade Laboral & Sim & Não & & \\
& $42(20 \%)$ & $168(80 \%)$ & & \\
Aposentadoria & Sim & Não & & \\
& $168(80 \%)$ & $42(20 \%)$ & $3.1-5.0 \mathrm{SM}$ & $\geq 5.1 \mathrm{SM}$ \\
Renda pessoal & $0.0-1.0 \mathrm{SM}$ & $1.1-3.0 \mathrm{SM}$ & $9(4,28 \%)$ & $3(1,42 \%)$ \\
(em faixas de SM) & $130(61,90 \%)$ & $68(32,38 \%)$ & $3.1-5.0 \mathrm{SM}$ & $\geq 5.1 \mathrm{SM}$ \\
Renda familiar & $0.0-1.0 \mathrm{SM}$ & $1.1-3.0 \mathrm{SM}$ & $19(9,04 \%)$ & $16(7,61 \%)$ \\
(em faixas de SM) & $44(20,95 \%)$ & $131(62,38 \%)$ & & \\
\hline
\end{tabular}

com sete pontos, variando de $\mathbf{1}$ (Discordo totalmente) a $\mathbf{7}$ (Concordo totalmente) (Aquino, 2009).

Inventário de Eventos de Vida Estressantes para Idosos (ELSI). Foi elaborado por Aldwin (1990), estando composto por 31 itens que apresentam eventos estressantes potencialmente vivenciados no ano anterior à coleta dos dados. Este instrumento avalia a frequência do acontecimento dos eventos estressantes e o nível de estresse atribuído pelo respondente. Neste sentido, as respostas são dadas em escala de seis pontos, variando de $\mathbf{0}$ (Evento não aconteceu) a 5 (Extremamente estressante). Sua análise é feita em função da soma dos seguintes atributos: (a) número de eventos estressantes $(\mathbf{0}=N a \tilde{o}, \mathbf{1}$ a $\mathbf{5}=\operatorname{Sim})$ e (b) soma do nível de estresse atribuído a todos os itens (soma das pontuações de 1 a 5). Tavares (2004) realizou a adaptação brasileira deste inventário, incluindo o item "Assumir mais responsabilidade com os filhos" e modificando "Morte de um neto" para "Morte do pai ou da mãe". Portanto, a versão considerada neste estudo foi esta última, formada por 32 itens, mantendo-se a escala de resposta.

Mini-Exame do Estado Mental (MEEM). Consiste em 30 itens que avaliam sete categorias de funções cognitivas: orientação temporal, orientação espacial, memória imediata, atenção e cálculo, evocação atrasada de palavras, linguagem e praxia construtiva (Folstein, Folstein, $\&$ McHugh, 1975). Sua pontuação total pode atingir 30 pontos; as notas de corte utilizadas para exclusão com base neste instrumento foram baseadas nos critérios da Rede FIBRA, que utilizou as médias menos um desvio padrão dos grupos de escolaridade do trabalho de Brucki,
Nitrini, Caramelli, Bertolucci e Okamoto (2003): 17 para os analfabetos, 22 para idosos com escolaridade entre 1 e 4 anos, 24 para os com escolaridade entre 5 e 8 anos e, por fim, 26 para aqueles que tinham 9 ou mais anos de escolaridade.

Questionário Demográfico. Foram incluídas perguntas de natureza demográfica na parte final do protocolo, visando descrever as características dos participantes do estudo. Concretamente, as seguintes perguntas foram tidas em conta: idade, sexo, estado civil, escolaridade e renda mensal do idoso.

\section{Procedimento}

Em atenção aos critérios estabelecidos pela Resolução 196/96, do Conselho Nacional de Saúde, este estudo foi realizado após parecer favorável do Comitê de Ética em Pesquisa (Protocolo $\mathrm{n}^{\circ}$ 0022.0.133.000-10), da Universidade Estadual da Paraíba (UEPB). A coleta de dados ocorreu entre os meses de junho e dezembro de 2010. Oito estudantes de graduação em Psicologia, de uma instituição de ensino público na Paraíba, foram previamente treinados para coletar os dados. Estes se dividiram em quatro duplas com o fim de percorrer os domicílios dos idosos e aplicar os instrumentos. Na ocasião foram descritos os objetivos do estudo e, em seguida, os idosos eram solicitados a participar. Após sua concordância em colaborar, recebiam o Termo de Consentimento Livre e Esclarecido (TCLE), que deveria ser assinado por eles ou um responsável. Inicialmente, procedeu-se a avaliação de seu status cognitivo mediante um teste de rastreio cognitivo (MEEM). Os idosos 
Melo, R. L. P., Eulálio, M. C., Gouveia, V. V. \& Silva, H. D. M. (2013). O Efeito do Estresse na Qualidade de Vida de Idosos: O Papel Moderador do Sentido de Vida.

que pontuaram abaixo da nota de corte para seu nível de escolaridade foram excluídos; esta decisão foi necessária para assegurar maior confiabilidade das respostas dos participantes. Os idosos excluídos receberam explicações compatíveis, sendo agradecidos por sua disponibilidade em colaborar; os demais responderam a todos os outros instrumentos anteriormente descritos. Nestes casos, um tempo médio de 50 minutos foi suficiente para concluir sua participação no estudo.

\section{Análise de Dados}

Utilizou-se o PASW (versão 19) para tabulação e análise dos dados. Foram calculadas análises estatísticas de distribuição de frequências, mediadas de tendência central e dispersão para as variáveis qualidade de vida, sentido de vida e estresse. Para a escala de Qualidade de vida e estresse foram utilizados os critérios estabelecidos pelos trabalhos de validação. Para a escala de sentido de vida (PilTest-12), devido à carência de estudos que utilizaram a escala, foi realizada uma análises de componentes principais. Também foram realizadas correlações de Pearson, correlações Bisseriais por ponto dos escores das escalas com os dados sociodemográficos. Para conhecer o papel moderador do sentido de vida foram utilizadas duas regressões lineares simples tendo como variável independente o sentido de vida e o estresse e como variável resposta a qualidade de vida. Posteriormente, realizou-se outra regressão linear simples de um termo interativo do sentido de vida e estresse com a qualidade de vida e por fim duas regressões lineares do estresse na qualidade de vida para cada grupo de sentido de vida (alto SV e baixo SV).

\section{Resultados}

Os resultados deste estudo são organizados de forma hierárquica, segundo a ordem de precedência para conhecer o papel moderador do sentido de vida em relação ao estresse e à qualidade de vida. Portanto, inicialmente, checou-se os parâmetros do instrumento PilTest-12. Posteriormente, procurou-se descrever as pontuações dos idosos em cada uma das medidas empregadas: qualidade de vida (WHOQOL-OLD), sentido de vida (PilTest-12) e estresse (ELSI). Depois, comprovaram-se as correlações das variáveis demográficas com indicadores de qualidade de vida, estresse e sentido de vida. Por fim, averiguou-se o papel moderador do sentido de vida em relação à influência do estresse na qualidade de vida.

\section{Parâmetros das Medidas de Qualidade e Sentido de Vida}

Procurou-se checar se a matriz de correlações inter-itens do Teste de Propósito de Vida era fatorializável, o que foi confirmada $[\mathrm{KMO}=0,81 \mathrm{e}$ Teste de Esfericidade de Bartlett, $\left.\chi^{2}(66)=447,18, p<0,001\right]$. Por meio de análise de componentes principais foi possível identificar dois componentes com valores próprios superiores a 1 , os quais explicaram conjuntamente $39,8 \%$ da variância total. Eles foram identificados como vazio existencial e realização existencial, apresentando índices de consistência interna de 0,73 e 0,63 , respectivamente. Um fator geral, incluindo todos os itens, apresentou índice de consistência interna adequado $(\alpha=0,77)$, sendo nomeado como sentido de vida.

\section{Descrição dos Indicadores de Qualidade de Vida, Sentido de Vida e Estresse}

Procurou-se descrever as pontuações dos participantes em cada um dos instrumentos utilizados, sendo os resultados apresentados na Tabela 1. É importante destacar que a classificação das pontuações foi realizada com base na divisão em três partes iguais da variação de cada escala. Assim, por exemplo, o instrumento PilTest-12 possui uma variação de 84 pontos, que foram divididos em três partes iguais correspondendo aos tercís respectivos.

Quanto ao WHOQOL-OLD, a maioria dos idosos considerou sua Qualidade de Vida Geral (QV Geral) como "boa ou muito boa" $(59,9 \% ; M=66,9, D P=10,97)$. As facetas Funcionamento dos Sentidos e Morte e Morrer apresentaram, respectivamente, $72,4 \%$ e $61,9 \%$ da amostra avaliando-as como "boa ou muito boa". No que se refere aos demais domínios, a Autonomia apresentou o maior percentual $(12,1 \% ; M=60,1, D P=16,39)$ da amostra avaliando-a como "ruim ou muito ruim", seguida de Intimidade $(7 \% ; M=67,6, D P=16,80)$ e Participação Social $(6,8 \% ; M=59,9, D P=16,09)$. O domínio Atividades Passadas, Presentes e Futuras foi o que apresentou maior percentual da amostra no tercil intermediário ("nem ruim nem boa"; 47,6\%; $M=64,7, D P=15,92$ ).

No que se refere ao Sentido de Vida (SV), a maioria dos idosos deste estudo (93\%; $M=65,2 ; D P=10,77)$ apresentou níveis altos. Coerentemente, eles também apresentaram nível alto de Realização Existencial (58,2\%; $M=35,6, D P=5,28)$ e baixo de Vazio Existencial $(68,7 \%$; $M=18,1, D P=7,84$ ).

Finalmente, quanto aos níveis de estresse, $61 \%$ dos idosos relataram ter vivenciado eventos nada ou pouco estressantes. No entanto, é valido ressaltar que 39\% apresentaram nível mediano ou alto de estresse. Os eventos mais frequentes foram "a morte de um amigo", estando presente em 119 participantes $(56,9 \%)$, seguido por "perda de memória" e "doença ou queda", ambos presentes em 96 idosos (45,9\%). Tiveram ainda 172 relatos ligados a morte de entes queridos, como pais, filhos e parentes próximos.

\section{Correlatos Demográficos de Qualidade de Vida, Sentido} de Vida e Estresse

Conhecida a adequação dos instrumentos de interesse, passou-se a avaliar em que medida suas pontuações estariam correlacionadas com nove variáveis demográficas: sexo $(\mathbf{1}=$ Masculino, $\mathbf{0}=$ Feminino $)$, estado civil $(\mathbf{1}=$ Casado, $\mathbf{0}=$ Não casado $)$, exerce atividade laboral $(\mathbf{1}=$ $\operatorname{Sim}, \mathbf{0}=$ Não $)$, está aposentado $(\mathbf{1}=\operatorname{Sim}, \mathbf{0}=$ Não $)$, nível escolar $(\mathbf{0}=$ Analfabeto, $\mathbf{1}=$ Estudos Básicos, $\mathbf{2}=$ Estudos Médios, $\mathbf{3}=$ Estudos Superiores $)$, vive sozinho $(\mathbf{1}=\operatorname{Sim}, \mathbf{0}=$ 
Tabela 2

Estatísticas Descritivas para as Medidas de Qualidade de Vida, Sentido de Vida e Estresse

\begin{tabular}{|c|c|c|c|c|c|}
\hline \multirow[b]{2}{*}{ Facetas } & \multicolumn{5}{|c|}{ WHOQOL-OLD } \\
\hline & $\begin{array}{l}\text { Ruim ou } \\
\text { muito ruim }\end{array}$ & $\begin{array}{c}\text { Nem ruim, } \\
\text { nem boa }\end{array}$ & $\begin{array}{l}\text { Boa ou } \\
\text { muito boa }\end{array}$ & $M(D P)$ & $M d n$ \\
\hline Funcionamento dos sentidos & $8,2 \%$ & $19,2 \%$ & $72,4 \%$ & $75,9(20,89)$ & 81 \\
\hline Autonomia & $12,1 \%$ & $51,7 \%$ & $36,1 \%$ & $60,1(16,39)$ & 63 \\
\hline Atividades (passado, presente e futuro) & $5,3 \%$ & $47,6 \%$ & $47,1 \%$ & $64,7(15,92)$ & 63 \\
\hline Participação social & $6,8 \%$ & $38,8 \%$ & $45,4 \%$ & $65,9(16,09)$ & 69 \\
\hline Morte e morrer & $14,9 \%$ & $2,4 \%$ & $61,1 \%$ & $67,9(23,42)$ & 75 \\
\hline Intimidade & $7,0 \%$ & $34,6 \%$ & $58,6 \%$ & $67,9(16,80)$ & 69 \\
\hline \multirow[t]{2}{*}{ QV - Pontuação total } & $1,0 \%$ & $39,1 \%$ & $59,9 \%$ & $66,9(10,97)$ & 68 \\
\hline & \multicolumn{5}{|c|}{ PilTest-12 } \\
\hline Fatores & $\begin{array}{l}\text { Baixo ou } \\
\text { muito baixo }\end{array}$ & Médio & $\begin{array}{c}\text { Alto ou } \\
\text { muito alto }\end{array}$ & $M(D P)$ & $M d n$ \\
\hline Vazio existencial & $68,7 \%$ & $28,4 \%$ & $2,8 \%$ & $18,1(7,84)$ & 16 \\
\hline Realização existencial & $1,9 \%$ & $39,9 \%$ & $58,2 \%$ & $35,6(5,28)$ & 36 \\
\hline \multirow[t]{2}{*}{ Sentido de vida } & $0,0 \%$ & $7,0 \%$ & $93,0 \%$ & $65,2(10,77)$ & 69 \\
\hline & \multicolumn{5}{|c|}{ ELSI } \\
\hline Pontuação total de Intensidade & $\begin{array}{c}\text { Nada ou } \\
\text { pouco } \\
\text { estressante }\end{array}$ & $\begin{array}{c}\text { Medianamente } \\
\text { estressante }\end{array}$ & $\begin{array}{c}\text { Muito ou } \\
\text { extremamente } \\
\text { estressante }\end{array}$ & $M(D P)$ & $M d n$ \\
\hline Estresse & $61,0 \%$ & $34,2 \%$ & $4,8 \%$ & $15,5(11,23)$ & 13 \\
\hline
\end{tabular}

Não), renda pessoal e renda familiar (ambas informadas em termos de moeda corrente, Real). Portanto, descrevem-se a seguir os padrões de correlações por construto avaliado, excetuando o estresse, que não apresentou qualquer relação com tais variáveis:

Qualidade de Vida. Quatro das variáveis demográficas não apresentaram qualquer correlação significativa com este construto $(p>0,05)$ : sexo, aposentadoria, nivel escolar e renda pessoal. Sua pontuação total foi correlacionada unicamente com o estado civil, com os casados apresentando maior pontuação $(r=0,14, p<$ $0,05)$; esta mesma variável se correlacionou com outros dois fatores específicos desta medida: morte e morrer $(r$ $=0,14, p<0,05)$ e intimidade $(r=0,29, p<0,001)$. A variável idade se correlacionou inversamente com $a u$ tonomia $(r=-0,17, p<0,05)$, enquanto que trabalha se correlacionou diretamente com este fator $(r=0,21, p<$ $0,01)$ e viver sozinho o fez inversamente com intimidade $(r=-0,16, p<0,05)$.

Sentido de Vida. A pontuação geral desta medida se correlacionou unicamente com o nivel escolar, fazendo-o de forma direta $(r=0,23, p<0,01)$, isto é, os idosos com maior escolaridade apresentaram maior sentido de vida. De modo coerente, seu fator específico denominado vazio existencial se correlacionou negativamente com o nível escolar $(r=-0,27, p<0,001)$; entretanto, não se observou correlação significativa com a realização existencial $(r=$ $0,12, p>0,05)$.

\section{Sentido de Vida como Variável Moderadora}

Com o fim de verificar se o SV moderaria o efeito do estresse na pontuação geral da qualidade de vida nos idosos, seguiram-se dois passos. Primeiramente, comprovou-se se o SV e o estresse predizem esta pontuação de qualidade de vida. Posteriormente, em caso afirmativo, procurou-se averiguar se a predição do estresse em relação à qualidade de vida se alteraria na presença de níveis diferentes de SV, isto é, baixo e alto. No primeiro caso, foram realizadas duas análises de regressão simples; a primeira tendo como preditor o SV e a segunda o estresse, sendo a qualidade de vida a variável dependente em ambos os casos. O SV explicou $24 \%$ da variabilidade de resposta para a pontuação geral de qualidade de vida. Por outro lado, o estresse também se mostrou um preditor adequado desta pontuação, contribuindo com a explicação de $14 \%$ da variabilidade de respostas.

No segundo passo, buscou-se avaliar se o SV poderia moderar a relação entre o estresse e a qualidade de vida. Portanto, foi analisado em que medida as linhas de regressão (as inclinações, os slopes) entre tais variáveis 
Melo, R. L. P., Eulálio, M. C., Gouveia, V. V. \& Silva, H. D. M. (2013). O Efeito do Estresse na Qualidade de Vida de Idosos: O Papel Moderador do Sentido de Vida.

diferiam significativamente para pessoas com baixa e alta pontuações em SV. Neste sentido, primeiramente foi inserida a interação entre estresse e SV (i.e., criou-se um termo de interação ao multiplicar o estresse pelo SV), com os resultados demonstrando que esta interação foi um preditor significativo da pontuação total em qualidade de vida $[\beta=-0,27 ; t(176)=-3,65, p<0,001]$. Isso sugeriu um efeito moderador do SV na relação entre estresse e qualidade de vida.

Com o propósito de conhecer o sentido da moderação previamente identificada, dividiu-se os idosos em categorias segundo sua amplitude no SV. Em virtude da primeira classificação do SV, por meio da variação da escala, não ter apresentado idosos no tercil com baixo sentido de vida, optou-se por uma nova classificação baseada na amplitude das pontuações do PilTest-12 em três partes iguais. Assim, considerando que o valor mínimo alcançado pela escala PilTest-12 foi 33 e o máximo foi 84 e que a amplitude foi de 51, formaram-se tercís de 17 pontos a partir do valor mínimo alcançado. Em seguida, foi excluído o grupo intermediário, restando os participantes com pontuações extremas: os que apresentavam baixa e alta pontuação em SV.

Posteriormente, realizou-se uma regressão linear simples do estresse na pontuação total de qualidade de vida para cada grupo de SV (baixo e alto). Os resultados demonstraram que a predição do estresse e qualidade de vida era significativa para os idosos com baixo $\mathrm{SV}[\beta=$ $-0,27 ; t(64)=-2,10, p<0,05]$, porém não o foi para os de alto $\mathrm{SV}[\beta=-0,08 ; t(60)=-0,63 ; p>0,05]$; a representação gráfica destes resultados é apresentada na Figura 1.

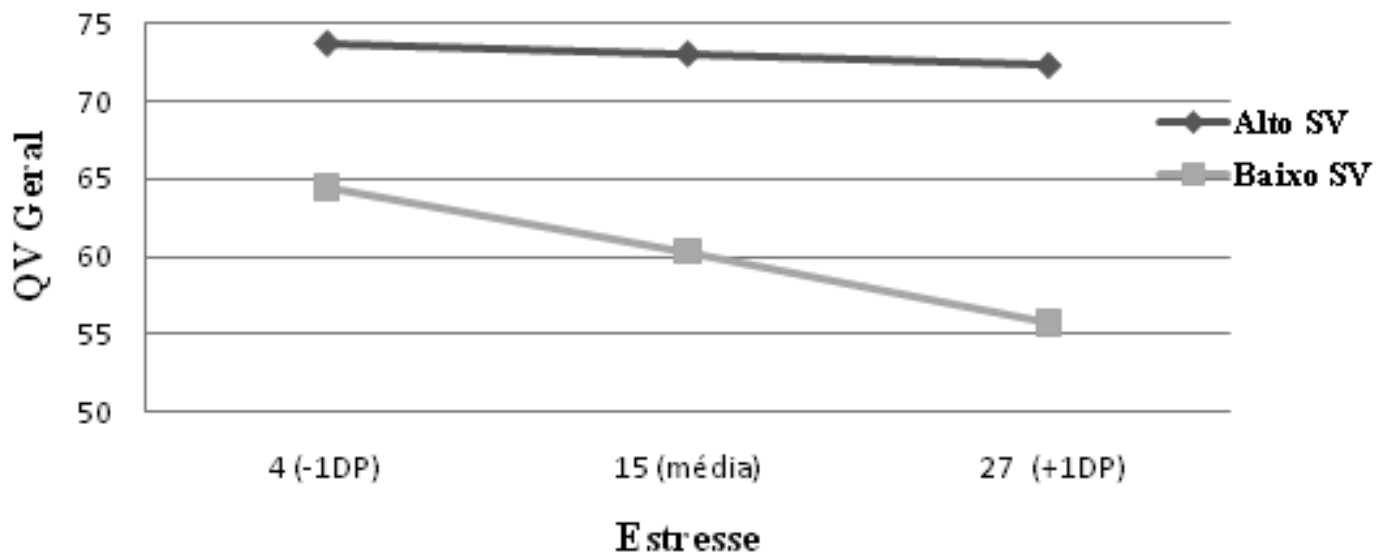

Figura 1. Efeito moderador do SV na relação entre o Estresse e a QV Geral

De acordo com estes resultados, a qualidade de vida dos idosos com nível baixo de sentido de vida (baixo SV) foi mais vulnerável ao estresse do que a daqueles que apresentaram nível alto desta variável (alto SV).-

\section{Discussão}

Este trabalho possuiu como objetivo principal examinar se o sentido de vida poderia atuar como recurso capaz de diminuir os efeitos do estresse na qualidade de vida de uma amostra de idosos residentes na cidade de Campina Grande - PB. As estatísticas descritivas demonstram que uma pequena parcela de idosos apresentou índices negativos de QV. Em relação aos índices de SV, estes foram majoritariamente positivos. Para os índices de Estresse, a maioria relatou ter vivenciado eventos nada ou pouco estressantes. Os eventos estressantes mais presentes na amostra foram respectivamente: morte de um amigo; problemas com a memória; e doença ou queda. Os escores de QV correlacionaram-se com o estado civil, viver sozinho, exercer atividade laboral e idade. O SV correlacionou-se unicamente com a escolaridade. O estresse não apresentou correlação com as variáveis sociodemográficas. Por fim, os resultados indicaram que a QV Geral dos idosos que apresentaram nível elevado de SV ficou menos vulnerável as alterações do Estresse.

\section{Indicadores de Qualidade de Vida, Sentido de Vida e Estresse}

Os resultados anteriormente descritos sobre o nível de qualidade de vida em idosos são coerentes com estudos prévios, encontrando-se no intervalo do que tem sido observado. Por exemplo, em estudo realizado em hospital escola de Natal (RN), Maués, Paschoal, Jaluul, França e Jacob (2010) encontraram médias de Qualidade de Vida Geral maiores que as do presente estudo $(M=83,7, D P=$ 11,92). No entanto, Torres, Reis, Reis e Fernandes (2009), trabalhando com idosos dependentes funcionalmente, encontraram médias bem inferiores $(M=47,9, D P=$ $33,40)$, tendo estes achados sido coerentes com aqueles descritos por Nunes, Menezes e Alchieri (2010), os quais trataram com idosos institucionalizados $(M=52,9, D P=$ 
17,70). Possivelmente, tais discrepâncias têm múltiplas explicações, porém duas plausíveis são: (a) a multiplicidade e subjetividade do construto qualidade de vida, que representa uma síntese de múltiplas determinações (Fleck et al., 2006); e (b) a natureza diversa das amostras tidas em conta. Pois, apesar de todos serem idosos, desfrutam de indicadores sociais e econômicos variados, pertencendo a contextos culturais e/ou institucionais diferentes. Contudo, estudos futuros serão necessários para dirimir dúvidas e avaliar estas duas explicações.

Quanto aos índices elevados de SV encontrados neste estudo, é possível que sejam respaldados em Reker (2001). Este autor afirma que, possivelmente, os idosos se constituem em um grupo com maior facilidade de desenvolver $\mathrm{SV}$ se comparados com adultos e jovens, uma vez que parecem ser mais realistas em relação a suas metas e têm menor predisposição ao vazio existencial. Portanto, por sua experiência de vida, é provável que as pessoas idosas tendam a fazer uma leitura mais autêntica da realidade e de suas capacidades, tomando decisões mais compatíveis com a situação e ficando menos expostos à frustração. Destaca-se, igualmente, que em virtude da escassez de estudos com idosos que tenham respondido o PilTest-12, oferecem-se com esta pesquisa pontuações que podem servir como referentes para avaliar o sentido de vida deste grupo etário com respeito a dita medida.

No tocante aos eventos estressantes, assim como ocorreu neste estudo, outros autores evidenciaram como eventos mais citados as questões ligadas à memória, saúde e finitude da vida (Aldwin, Sutton, \& Lachman, 1996; Chapleski, Kaczynski, Gerbi, \& Lichtenberg, 2004; Couto, 2007; Fortes-Burgos et al., 2008).

Problemas graves de saúde e morte são situações que os indivíduos podem percebê-las como inevitáveis, sendo vividas com um grau elevado de emoções negativas, sentimentos de frustração e desamparo, o que potencializa a sensação de falta de controle em relação à vida (Schulz \& Heckhausen, 1998). Neste ponto cabe indicar que, talvez, a preocupação com morte não esteja ligada ao evento em si, mas ao medo de sofrimento que possa anteceder o processo de morte (Lunardi, Sulzbach, Nunes, $\&$ Lunardi, 2001). Acrescenta-se, a este contexto, o fato de seus amigos e familiares estarem morrendo, o que pode aumentar a percepção do evento como um processo doloroso, potencializando sentimentos de insegurança, tristeza e insatisfação (Frumi \& Celich, 2006). No entanto, mesmo diante dessas situações, Frankl (2003) entende que é possível transformar uma tragédia em uma conquista humana, para isso lança mão do conceito de valor de atitude, como um valor capaz de dar sentido à vida. Para Frankl (1978) o sofrimento, quando rico de significado, pode transformar-se em desempenho e auxiliar o homem a enxergar outras possibilidades frente às desventuras inevitáveis que se apresentam ao longo da vida. Oferecendo subsídio na forma como se percebe a morte, não ficando apenas com a sensação que pouco tem o que se fazer diante do problema.

\section{Correlatos Demográficos de Qualidade de Vida}

É válido salientar que os correlatos das variáveis demográficas com a Qualidade e o Sentido de Vida, variaram de $r=0,12$ a $r=0,29$, o que para Cohen (1988) são correlações de efeito pequeno $( \pm 0,1)$ e médio $( \pm 0,3)$.

Os idosos casados apresentaram maiores pontuações na QV Geral e nas facetas Morte e Morrer e Intimidade, enquanto os que relataram morar só apresentaram menor pontuação em intimidade. Alguns estudos mostraram que a maioria dos homens e mulheres que mantém algum tipo de vínculo conjugal apresenta mais sentimentos de companheirismo e amor, pois expressam melhor seus afetos e a sexualidade, mesmo que seja por meio de simples gesto, como um olhar, um abraço, um toque de mão ou um sorriso (Moura, Leite, \& Hildebrand, 2008; Perry \& Potter, 2005). Isso pode potencializar a qualidade de vida, a sensação de prazer em viver. Nesta direção, E. L. Garcia, Banegas, Perez-Regadera, Cabrera e Rodriguez-Artalejo (2005), em estudo populacional com 4.000 idosos da Espanha, concluíram que aqueles idosos que não moram só possuem melhor qualidade de vida, pois parecem possuir melhor suporte emocional e sensação de segurança decorrente de um senso de pertencimento e integração.

As correlações indicam que os idosos com maior idade tendem a apresentar menor pontuação na Faceta Autonomia. Os sujeitos com maior autonomia possuem mais sentimentos de controle sob sua vida, isso gera a possibilidade de fazer planos para seu futuro o que o torna mais esperançoso, melhorando sua qualidade de vida (Agich, 2008). Além da idade, o declínio da autonomia pode ocorrer devido a limitações físicas, sociais, financeira ou pela impossibilidade de trabalhar (Bulla \& Kaefer, 2003). Cabe aqui ressaltar o papel fundamental do trabalho para o desenvolvimento pessoal e reconhecimento social, servindo como um regulador da organização social (Zanelli \& Silva, 1996). Em linha com estes autores, em estudo populacional com idosos que vivem em regiões metropolitanas no Brasil, Lima-Costa, Guerra, Barreto e Maia (2000) observaram que o trabalho estava associado com maior independência nas esferas física, social e econômica da vida, sendo um preditor importante de saúde destas pessoas.

Finalmente, no caso do sentido de vida, os idosos com maior nível educacional apresentaram maiores pontuações. Embora não tenham sido encontrados estudos e subsídios teóricos que pudessem explicar esta relação, uma conjetura pode ser apresentada: as pessoas com maiores pontuações em sentido de vida apresentam maior abertura à mudança, uma visão mais universal do mundo, não se limitando a aspectos restritos da vida, sendo mais orientadas a valores intrínsecos, interativos ou suprapessoais (Aquino, 2009), o que parece ser compatível com pessoas que apresentam maior escolaridade. Entretanto, estudos futuros poderão contribuir para entender esta relação, indicando, se for o caso, as vantagens de educar os idosos. 
Melo, R. L. P., Eulálio, M. C., Gouveia, V. V. \& Silva, H. D. M. (2013). O Efeito do Estresse na Qualidade de Vida de Idosos: O Papel Moderador do Sentido de Vida.

\section{Sentido de Vida como Variável Moderadora}

Alguns autores discutem a capacidade que o SV tem de auxiliar as pessoas a enfrentar eventos estressantes. Melton e Schulenberg (2008) colocam que os idosos com maiores níveis de SV são emocionalmente mais estáveis, mais criativos, investem mais tempo em atividades que envolvem a família e os amigos e se percebem com mais controle de eventos estressantes. Isso auxilia na ampliação da autoconfiança e da uma visão mais positiva e otimista do mundo (Ho et al., 2010). No presente trabalho o evento estressante mais presente foi a morte de um(a) amigo(a), diante disso Baumeister e Vohs (2002) argumentam que idosos que apresentam melhores níveis de SV lidam melhor com o luto de entes queridos, pois conseguem atribuir um significado a perda.

Frankl $(2003,2004)$ propõe que o SV tende a atuar como um efeito de tampão ou deslocamento das consequência deletérias das dificuldades no bem-estar do indivíduo. Dessa forma, a percepção de que o sofrimento tem um sentido faria com que as dificuldades impostas pelos eventos estressantes fossem encaradas como um desafio compreensivo e manejável. Para S. C. Garcia (2008) e Schulenberg e Melton (2010) a procura deste sentido é um alicerce da motivação para a pessoa construir resiliência. É importante assinalar que alguns autores têm se dedicado a desenvolver intervenções pautadas nos pressupostos da Logoterapia, procurando minimizar os efeitos danosos do estresse na saúde dos indivíduos. Fabry $(1985,1988)$ relata procedimentos individuais e de grupo para iniciar reflexões acerca desse sentido, agrupados em cinco caminhos: Auto conhecimento, possibilidades de escolha, nossa singularidade, responsabilidade a auto-transcedência. Para Melton e Schulenberg (2008) a logoterapia pode ser integrada a outras formas de psicoterapia e estratégias de tratamento, tais como gestão de medicamentos, podendo acomodar uma grande variedade de tratamentos clínicos.

\section{Conclusão}

Os resultados sugerem que o SV desempenha uma função moderadora da relação do estresse na QV Geral dos idosos, atuando como um recurso protetor. Intervenções reguladas pelos pressupostos teóricos da Logoterapia podem ser uma importante ferramenta para trabalhar a Qualidade de Vida e saúde dos idosos. Por fim, será fundamental seguir investigando neste âmbito da qualidade de vida em idosos. Identificar fatores que a promovam, mesmo diante de eventos estressantes que são inevitáveis e inerentes à terceira idade, deveria se constituir em uma agenda de pesquisa. Parece pouco produtivo dirigir o olhar para os fatores de risco da qualidade de vida. É importante pensar em fatores que assegurem sua proteção. Neste âmbito, as relações interpessoais podem ser um fator importante a considerar. Um desafio poderia ser estudar estilos de socialização filiais. Parece paradoxal, mas as pessoas são (re)socializadas em múltiplas etapas de suas vidas, e na terceira idade não é diferente; os filhos, comumente, têm um papel decisivo, oferecendo amor, mas também alguma disciplina, evitando prejuízos para a convivência familiar. Provavelmente, a qualidade de vida experimentada pelos idosos compartilhe alguma variância com a percepção dos estilos filiais.

\section{Referências}

Agich, G. J. (2008). Dependência e autonomia na velhice: Um modelo ético para o cuidado de longo prazo. São Paulo, SP: Edições Locais.

Aldwin, C. M. (1990). The Elders Life Stress Inventory: Egocentric and nonegocentric stress. In M. A. P. Stephens, J. H. Crowther, S. E. Hobfoll, \& D. L. Tennenbaum (Eds.), Stress and coping in later-life families (pp. 49-69). New York: Hemisphere.

Aldwin, C. M., \& Gilmer, D. F. (2004). Health, illness, and optimal aging: Biological and psychological perspectives. Thousand Oaks, CA: Sage.

Aldwin, C. M., Sutton, K. J., \& Lachman, M. (1996). The development of coping resources in adulthood. Journal of Personality, 64, 837-871.

Aquino, T. A. A. (2009). Atitudes e intenções de cometer suicídio: Seus correlatos existenciais e normativos (Tese de doutorado, Departamento de Psicologia, Universidade Federal da Paraíba, João Pessoa, PB, Brasil).

Banco Internacional para a Reconstrução e o Desenvolvimento. (2011). Envelhecendo em um Brasil mais velho. Brasília, DF: Banco Mundial.

Bauer-Wu, S., \& Farran, C. J. (2005). Meaning in life and psychospiritual functioning: A comparison of breast cancer survivors and healthy women. Journal of Holistic Nurse, 23, 172-190.

Baumeister, R. F., \& Vohs, K. D. (2002). The pursuit of meaningfulness in life. In C. R. Snyder \& S. J. Lopez (Eds.), Handbook of positive psychology (pp. 608-618). NewYork: Oxford University Press.

Brucki, S. M. D., Nitrini, R., Caramelli, P., Bertolucci, P. H. F., \& Okamoto, I. H. (2003). Sugestões para o uso do Mini-Exame do Estado Mental no Brasil. Arquivos de Neuropsiquiatria, 61, 777-781.

Bulla, L. C., \& Kaefer, C. O. (2003). Trabalho e aposentadoria: As repercussões sociais na vida do idoso aposentado. Texto \& Contexto Enfermagem, 2, 34-46.

Chapleski, E. E., Kaczynski, R., Gerbi, S. A., \& Lichtenberg, P. A. (2004). American Indian elders and depression: Short- and long-term effects of life events. Journal of Applied Gerontology, 23, 40-57.

Cohen, J. (1988). Statistical power analysis for the behavioural sciences $\left(2^{\text {nd }}\right.$ ed.). New York: Academic Press.

Couto, M. C. P. P. (2007). Fatores de risco e de proteção na promoção de resiliencia no envelhecimento (Dissertação de mestrado, Departamento de Psicologia, Universidade Federal do Rio Grande do Sul, Porto Alegre, RS, Brasil).

Crumbaugh, J. H., \& Maholich, L. T. (1964). The psychometric approach to Frankl's concept of noogenic neurosis. Journal of Clinical Psychology, 20, 200-207.

Fabry, J. (1985). Wege zur Selbstfindung. Freiburg im Breisgau, Germany: Herderbücherei.

Fabry, J. (1988). Guideposts to meaning. Oakland, CA: New Harbinger.

Fleck, M. P. A., Chachamomovich, E., \& Trentin, C. (2006). Desenvolvimento e validação da versão em Português do módulo WHOQOL-OLD. Revista de Saúde Pública, 40, 785-910. 
Folstein, M., Folstein, S., \& McHugh, P. (1975). Mini-Mental State. A practical method for grading the cognitive status of patients for the clinician. Journal of Psychiatric Research, 12, 189-198.

Fortes-Burgos, A. C. G., Neri, A. L., \& Cupertino, A. P. F. B. (2008). Eventos estressantes, estratégias de enfrentamento, auto-eficácia e sintomas depressivos entre idosos residentes na comunidade. Psicologia: Reflexão e Crítica, 21, 74-82.

Frankl, V. E. (1978). Fundamentos antropológicos da psicoterapia. Rio de Janeiro, RJ: Zahar.

Frankl, V. E (2003). Psicoterapia e sentido da vida: Fundamentos da logoterapia e análise existencial (4. ed.). São Paulo, SP: Quadrante.

Frankl, V. E. (2004). Em busca de sentido (19. ed.). Petrópolis, RJ: Vozes.

Frumi, C., \& Celich K. L. S. (2006). O olhar do idoso frente ao envelhecimento e à morte. Revista Brasileira de Ciências do Envelhecimento Humano, 3, 92-100.

Garcia, E. L., Banegas, J. R., Perez-Regadera, A. G., Cabrera, R. H., \& Rodriguez-Artalejo, F. (2005). Social network and health related quality of line in older adults: A populationbased a study in Spain. Quality of Life Research, 14, 511-520.

Garcia, S. C. (2008). A resiliência no indivíduo especial: Uma visão logoterapêutica. Revista Educação Especial, 31, 25-36.

Ho, M. Y., Cheung, F. M., \& Cheung, S. F. (2010). The role of meaning in life and optimism in promoting well-being. Personality and Individual Differences, 48, 658-663.

Jim, H. S. L., \& Andersen, B. L. (2007). Meaning in life mediates the relationships between physical and social functioning and distress in cancer survivors. British Journal of Health Psychology, 12, 363-381.

Kashdan, T. B., \& Steger, M. F. (2007). Curiosity and pathways to well-being and meaning in life: Traits, states, and everyday behaviors. Motivation and Emotion, 31, 159-173.

Krause, N. (2009). Meaning in life and mortality. Journal of Gerontology: Social Sciences, 64(4), 517-527

Lazarus, R. S., \& Folkman, S. (1984). Stress, appraisal, and coping. New York: Springer.

Lima-Costa, M. F. F., Guerra, H. L., Barreto, S. M., \& Maia, R. (2000). Diagnóstico da situação de saúde da população idosa brasileira: Um estudo da mortalidade e das internações hospitalares públicas. Informe Epidemiológico do SUS, 9, 23-41.

Lunardi, W. D., Filho, Sulzbach, R. C., Nunes, A. C., \& Lunardi, V. L. (2001). Percepções e condutas dos profissionais de enfermagem frente ao processo de morrer e morte. Texto \& Contexto Enfermagem, 10,60-81.

Margis, R., Picon, P., Cosner, A. F., \& Silveira, R. O. S. (2003). Relação entre estressores, estresse e ansiedade. Revista de Psiquiatria do Rio Grande do Sul, 25, 65-74.

Mascaro, N., \& Rosen, D. H. (2005). Existential meaning's role in the enhancement of hope and prevention of depressive symptoms. Journal of Personality, 73, 985-1014.

Maués, C. R., Paschoal, S. M. P., Jaluul, O., França, C. C., \& Jacob, W., Filho (2010). Avaliação da qualidade de vida: Comparação entre idosos jovens e muito idosos. Revista Brasileira de Clínica Médica, 8, 405-410.

McKnight, P. E., \& Kashdan, T. B. (2009). Purpose in life as a system that creates and sustains health and well-being: An integrative, testable theory. Review of General Psychology, 13, 242-251.

Melton, A. M. A., \& Schulenberg, S. E. (2008). On the measurement of meaning: Logotherapy's empirical contributions to humanistic psychology. The Humanistic Psychologist, 36, 31-44.
Monat, A., Lazarus, R. S., \& Reevy, G. (2007). The Praeger handbook of stress and coping. Westport, CT: Praeger.

Moura, I., Leite, M. T., \& Hildebrand, L. M. (2008). Idosos e sua percepção acerca da sexualidade na velhice. Revista Brasileira de Ciências do Envelhecimento Humano, 5, 132-140.

Neri, A. L., \& Fortes, A. C. G. (2006). A dinâmica do estresse e enfrentamento na velhice e sua expressão no prestar cuidados a idosos no contexto da família. In E. V. Freitas, L. Py, F. A. X. Cançado, J. Doll, \& M. L. Gorzoni (Eds.), Tratado de Geriatria e Gerontologia (pp. 1277-1288). Rio de Janeiro, RJ: Guanabara Koogan.

Nunes, V. M. A., Menezes, R. M. P., \& Alchieri, J. C. (2010). Avaliação da qualidade de vida em idosos institucionalizados no município de Natal, estado do Rio Grande do Norte. Acta Scientiarum Health Sciences, 32, 119-126.

Park, C. L., Malone, M. R., Suresh, D. P., Bliss, D., \& Rosen, R. I. (2008). Coping, meaning in life, and quality of life in congestive heart failure patients. Quality of Life Research, 17, 21-26.

Perry, A. G., \& Potter, P. A. (2005). Grande tratado de Enfermagem Prática: Clínica e prática hospitalar (3. ed.). São Paulo, SP: Santos.

Reker, G. T. (1997). Personal meaning, optimism, and choice: Existential predictors of depression in community and institutional elderly. The Gerontologist, 37, 709-716.

Reker, G. T. (2001). Manual life attitude profile - Revised. Ontario, Canada: Student Psychologists Press.

Reker, G. T., \& Wong, P. T. P. (1988). Aging as an individual process: Toward a theory of personal meaning. In J. E. Birren \& V. L. Bengston (Eds.), Emergent theories of aging (pp. 214-246). New York: Springer.

Schulenberg, S. E., \& Melton, A. M. A. (2010). A confirmatory factor-analytic evaluation of the Purpose in Life Test: Preliminary psychometric support for a replicable two-factor model. Journal of Happiness Study, 11, 95-111.

Schulz, R., \& Heckhausen, J. (1998). Emotion and control: A life-span perspective. In P. Lawton (Ed.), Annual review of gerontology and geriatrics (Vol. 17, pp. 185-205). Philadelphia, PA: Springer.

Steger, M. F., Mann, J. R., Michels, P., \& Cooper, T. C. (2009). Meaning in life. In S. J. Lopez (Ed.), Handbook of positive psychology ( $2^{\text {nd }}$ ed., pp. 679-689). Oxford, UK: Oxford University Press.

Steger, M. F., \& Frazier, P. (2005). Meaning in life: One link in the chain from religiousness to well-being. Journal of Counseling Psychology, 52(4), 574-582.

Tavares, S. S. (2004). Sintomas depressivos entre idosos: Relações com classe, mobilidade e suporte social percebidos e experiência de eventos estressantes (Dissertação de mestrado, Departamento de Psicologia Educacional, Universidade de Campinas, SP, Brasil).

Torres, G. V., Reis, L. A., Reis, L. A., \& Fernandes, M. H. (2009). Qualidade de vida e fatores associados em idosos dependentes em uma cidade do interior da Bahia. Jornal Brasileiro de Psiquiatria, 58, 39-44.

Zanelli, J. C., \& Silva, N. (1996). Programa de preparação para aposentadoria. Florianópolis, SC: Insular. 